TRANSACTIONS OF THE

AMERICAN MATHEMATICAL SOCIETY

Volume 356, Number 10, Pages 3823-3839

S 0002-9947(04)03647-5

Article electronically published on May 11, 2004

\title{
HIGHER HOMOTOPY COMMUTATIVITY OF $H$-SPACES AND THE PERMUTO-ASSOCIAHEDRA
}

\author{
YUTAKA HEMMI AND YUSUKE KAWAMOTO \\ Dedicated to the memory of Professor Masahiro Sugawara
}

\begin{abstract}
In this paper, we give a combinatorial definition of a higher homotopy commutativity of the multiplication for an $A_{n}$-space. To give the definition, we use polyhedra called the permuto-associahedra which are constructed by Kapranov. We also show that if a connected $A_{p}$-space has the finitely generated mod $p$ cohomology for a prime $p$ and the multiplication of it is homotopy commutative of the $p$-th order, then it has the mod $p$ homotopy type of a finite product of Eilenberg-Mac Lane spaces $K(\mathbb{Z}, 1) \mathrm{s}, K(\mathbb{Z}, 2) \mathrm{s}$ and $K\left(\mathbb{Z} / p^{i}, 1\right) \mathrm{s}$ for $i \geq 1$.
\end{abstract}

\section{INTRODUCTION}

The notion of $H$-spaces was introduced to study Lie groups from a homotopy theoretic point of view. In recent decades, several theorems have been proved about the finite $H$-spaces (cf. [7] and [15]), which suggest that the finite $H$-spaces have many similar properties to those of the Lie groups.

Since being an $H$-space is a homotopy theoretic property, a space with the homotopy type of an $H$-space is also an $H$-space. The typical example of an $H$-space is a space $X$ of the homotopy type of a loop space $\Omega Y$ for some space $Y$. Sugawara 24] gave a criterion for a space to be of the homotopy type of a loop space. His criterion is a higher homotopy associativity of the multiplication. Later Stasheff 22] expanded the definition of Sugawara and reached the concept of the $A_{n}$-space. An $A_{n}$-space is by definition an $H$-space such that the multiplication is higher homotopy associative of the $n$-th order. The polyhedra used in his combinatorial definition are called the associahedra.

In 1960, Sugawara 25] also considered a higher homotopy commutativity of the multiplication of an associative $H$-space. Later Williams [26 considered another type of higher homotopy commutativity which is weaker than the one of Sugawara. In his combinatorial definition, Williams used polyhedra called the permutohedra which are originally introduced by Milgram [18] to construct approximations to the iterated loop spaces.

In the definitions by Sugawara and Williams, the multiplications of the spaces are assumed to be strictly associative. In this paper, we prove that we can define the

Received by the editors November 27, 2001.

2000 Mathematics Subject Classification. Primary 55P45, 55P48; Secondary 55P15, 52B11.

$K e y$ words and phrases. Higher homotopy commutativity, $H$-spaces, $A_{n}$-spaces, $A C_{n}$-spaces, permuto-associahedra. 

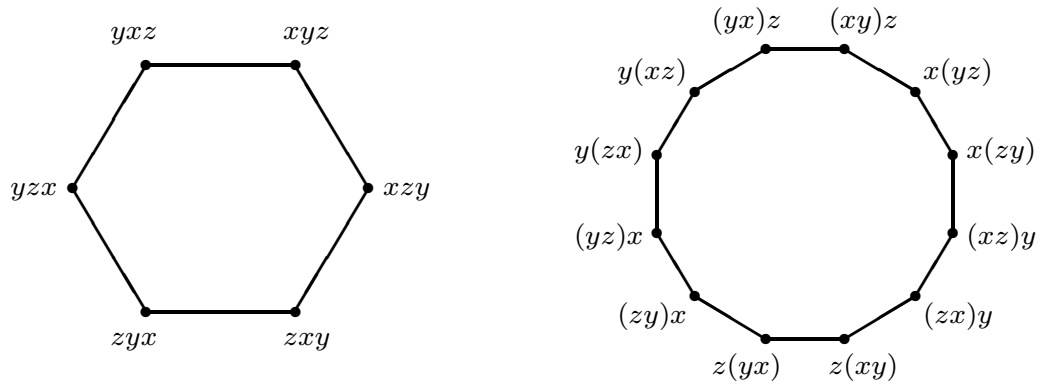

FIGURE 1. Higher homotopy commutativity of the third order

higher homotopy commutativity of the $n$-th order only assuming the multiplication is homotopy associative of the $n$-th order. For example, the higher homotopy commutativity of the second order is just the homotopy commutativity, so it does not need to assume any homotopy associativity on the multiplication. The higher homotopy commutativity of Williams of the third order is illustrated by the left hexagon in Figure 1. Thus, the definition is generalized for homotopy associative $H$-spaces by using the right dodecagon in Figure 1

Hemmi [5] considered a generalization of the higher homotopy commutativity of Williams in the case of $A_{n}$-spaces, and introduced the quasi $C_{n}$-space. However, his definition is not a combinatorial one. Moreover, the definition of the quasi $C_{n}$-space uses the projective spaces of the multiplication of the $A_{n}$-space (see $\S 3$ ). Since it is not known if the projective spaces are compatible with fibrations, the quasi $C_{n}$-space is not easy to handle. For example, the authors do not know if the covering spaces inherit the property of being a quasi $C_{n}$-space.

The reason why Hemmi gave such an artificial definition is that the polyhedra used in a proper combinatorial definition become very complicated since they should be given by combining the permutohedra and the associahedra.

In 1993, such polyhedra called the permuto-associahedra were constructed by Kapranov [8]. Due to his construction, a combinatorial definition of the higher homotopy commutativity has been possible now. In the present paper, we give the combinatorial definition. An $A_{n}$-space with a multiplication of this sort is called an $A C_{n}$-space (see Definition 3.1). From the definition, $X$ is an $A C_{2}$-space if and only if $X$ is a homotopy commutative $H$-space (see Example 3.2 (1)). Moreover, our definition coincides with the one of Williams if the multiplication of the given space is strictly associative (see Corollary 3.6).

According to Hemmi [5, Prop. 2.3], a homotopy commutative $H$-space is a quasi $C_{2}$-space, and if the multiplication is homotopy associative, then the converse also holds (see also [23, Thm. 13.6]). In the case of $A C_{n}$-spaces, we have the following result:

Theorem A. (1) If $X$ is an $A C_{n}$-space, then $X$ is a quasi $C_{n}$-space.

(2) If $X$ is an $A_{n+1}$-space having a quasi $C_{n}$-space structure, then $X$ is an $A C_{n^{-}}$ space.

Since $A C_{n}$-spaces are quasi $C_{n}$-spaces, the theorems for quasi $C_{n}$-spaces are also valid for $A C_{n}$-spaces. In particular, the $\bmod p$ torus theorems proved by Hemmi [5] and Kawamoto [11] are also true for $A C_{p}$-spaces. Besides, since the universal 
covering of an $A C_{n}$-space is also an $A C_{n}$-space (see Lemma 3.9), we have the following stronger version:

Theorem B. Let $p$ be a prime. If $X$ is a connected $A C_{p}$-space such that the mod $p$ cohomology $H^{*}(X ; \mathbb{Z} / p)$ is finitely generated as an algebra, then $X$ is $\bmod p$ homotopy equivalent to a finite product of Eilenberg-Mac Lane spaces $K(\mathbb{Z}, 1) s$, $K(\mathbb{Z}, 2) s$ and $K\left(\mathbb{Z} / p^{i}, 1\right) s$ for $i \geq 1$.

In the above theorem, the condition of $A C_{p}$-space cannot be relaxed to $A C_{p-1^{-}}$ space. In fact, the odd dimensional sphere $\left(S^{2 n-1}\right)_{p}^{\wedge}$ completed at $p$ is an $A C_{p-1^{-}}$ space for any $n \geq 1$ (see Proposition 3.8).

In the case of finite $A C_{p}$-spaces, we have the following corollary:

Corollary 1.1. Let $p$ be a prime. If $X$ is a connected finite $A C_{p}$-space, then $X$ is mod $p$ homotopy equivalent to a torus.

The above results are considered as mod $p$ versions of the torus theorems by Hubbuck 6], Lin [13, Slack 21], Lin-Williams 16] and Broto-Crespo [3. For the details of the $\bmod p$ torus theorems, see Aguadé-Smith [1, Hemmi [5], Kawamoto [9], [10], [11], Kawamoto-Lin [12, Lin [14] and McGibbon [17]. In particular, since the loop space of an $H$-space is an $A C_{n}$-space for any $n \geq 1$ (see Example $3.2(3)$ ), we have the following result:

Theorem 1.2 ([9, Thm. A]). Let $p$ be a prime. If $X$ is a simply connected mod $p$ $H$-space such that the mod $p$ cohomology $H^{*}(\Omega X ; \mathbb{Z} / p)$ is finitely generated as an algebra, then $\Omega X$ is mod $p$ homotopy equivalent to a finite product of Eilenberg-Mac Lane spaces $K(\mathbb{Z}, 1) s, K(\mathbb{Z}, 2) s$ and $K\left(\mathbb{Z} / p^{i}, 1\right)$ s for $i \geq 1$.

For the rest of this paper, all spaces are assumed to be completed at a prime $p$ in the sense of Bousfield-Kan 2]. An $H$-space which is completed at $p$ is called a $\bmod p H$-space, and it is called finite if its $\bmod p$ cohomology is finite dimensional.

This paper is organized as follows: In $\S 2$, we first recall the permuto-associahedra constructed by Kapranov [8. Then we show that the permutohedra are decomposed by using the associahedra and the permuto-associahedra (see Proposition [2.5). In $\S 3$, we give the combinatorial definition of the $A C_{n}$-form on an $A_{n}$-space by using the permuto-associahedra. Then we prove Theorem A by using the decompositions of the permutohedra in $\S 2$. By combining Theorem A with a result of Kawamoto [11] on quasi $C_{p}$-spaces with finitely generated $\bmod p$ cohomology, we give the proof of Theorem B.

\section{Permuto-Associahedra}

Stasheff [22] constructed a collection of special complexes $\left\{K_{n}\right\}_{n \geq 2}$ such that $K_{n}$ is homeomorphic to the $(n-2)$-dimensional ball for $n \geq 2$. He used the collection $\left\{K_{n}\right\}_{n \geq 2}$ to introduce the higher homotopy associativity of $H$-spaces (see $\S 3)$. The complex $K_{n}$ is called the $(n-2)$-dimensional associahedron for $n \geq 2$. Let $L_{n}=\partial K_{n}$. Then by [22, p. 278],

$$
L_{n}=\bigcup_{r, s, k} K_{k}(r, s)
$$

for $r, s \geq 2$ with $r+s=n+1$ and $1 \leq k \leq r$. The facet (codimension one face) $K_{k}(r, s)$ is homeomorphic to $K_{r} \times K_{s}$ by the face operator $\partial_{k}(r, s): K_{r} \times K_{s} \rightarrow$ $K_{k}(r, s)$ satisfying some relations (see [22, p. 278, 3(a),(b)]). Furthermore, there is 

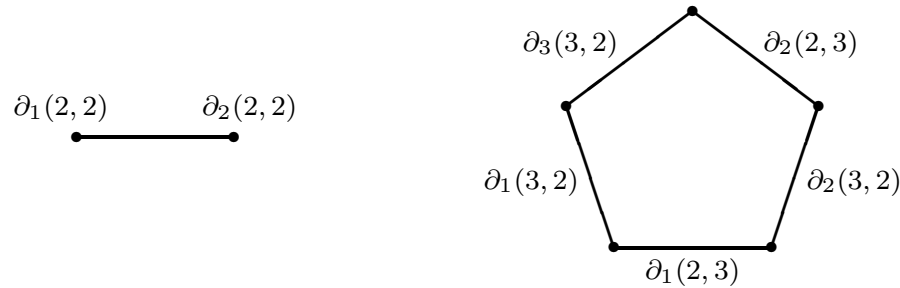

Figure 2. The associahedra $K_{3}$ and $K_{4}$

a collection of degeneracy operators $\left\{\theta_{j}: K_{n} \rightarrow K_{n-1}\right\}_{1 \leq j \leq n}$ satisfying suitable conditions (see [22, I, Prop. 3]). Here Figure 2] illustrates the associahedra $K_{3}$ and $K_{4}$.

Later Kapranov [8] constructed another collection of special complexes $\left\{\Gamma_{n}\right\}_{n \geq 1}$. By [8, Thm. 2.5], $\Gamma_{n}$ is homeomorphic to the $(n-1)$-dimensional ball for $n \geq 1$. The complex $\Gamma_{n}$ is closely related to the associahedra $\left\{K_{n}\right\}_{n \geq 2}$, and is called the $(n-1)$-dimensional permuto-associahedron for $n \geq 1$ (Kapranov [8] denoted the complex by $K P_{n}$ for $n \geq 1$ ). It is remarkable that Reiner-Ziegler [20, Thm. 2] reconstructed $\Gamma_{n}$ as the convex hull of a finite set of points in $\mathbf{R}^{n}$ for $n \geq 1$ (see also Ziegler [29, Example 9.14]).

Let $\mathbf{n}=(1, \ldots, n)$. For $l \geq 1$, we denote a subsequence of $\mathbf{n}$ of length $l$ by $\alpha=\left(a_{1}, \ldots, a_{l}\right)$. Let $\alpha: \mathbf{l} \rightarrow \mathbf{n}$ denote the composite $i_{\alpha} j_{\alpha}$, where $i_{\alpha}: \alpha \rightarrow \mathbf{n}$ is the inclusion and $j_{\alpha}: \mathbf{l} \rightarrow \alpha$ is the map defined by $j_{\alpha}(i)=a_{i}$ for $1 \leq i \leq l$. Let $t_{1}, \ldots, t_{m} \geq 1$ with $t_{1}+\cdots+t_{m}=n$. A partition of $\mathbf{n}$ of type $\left(t_{1}, \ldots, t_{m}\right)$ is an ordered sequence $\left(\alpha_{1}, \ldots, \alpha_{m}\right)$ consisting of disjoint subsequences $\alpha_{i}$ of length $t_{i}$ for $1 \leq i \leq m$ with $i_{\alpha_{1}}\left(\alpha_{1}\right) \cup \cdots \cup i_{\alpha_{m}}\left(\alpha_{m}\right)=\mathbf{n}$.

From the construction of $\Gamma_{n}$, there is a natural way to describe all the faces of it. By [29 Def. 9.13], a facet (codimension one face) of $\Gamma_{n}$ is represented by a partition $\left(\alpha_{1}, \ldots, \alpha_{m}\right)$ of $\mathbf{n}$ with $m \geq 2$, and a codimension two face is represented by inserting a pair of parentheses in a partition $\left(\alpha_{1}, \ldots, \alpha_{m}\right)$ as

$$
\left(\alpha_{1}, \ldots, \alpha_{i-1},\left(\alpha_{i}, \ldots, \alpha_{j}\right), \alpha_{j+1}, \ldots, \alpha_{m}\right)
$$

with $1 \leq i<j \leq m$. In general, a codimension $s+1$ face of $\Gamma_{n}$ is represented by inserting $s$ pairs of parentheses in a meaningful way to a partition $\left(\alpha_{1}, \ldots, \alpha_{m}\right)$ of $\mathbf{n}$ such that any pair of parentheses includes at least two elements each of which is $\alpha_{i}$ or a parenthesized sequence. In this manner, vertices of $\Gamma_{n}$ are represented by all meaningful complete ways of inserting parentheses to partitions of $\mathbf{n}$ of type $(1, \ldots, 1)$.

Now the facet of $\Gamma_{n}$ corresponding to a partition $\left(\alpha_{1}, \ldots, \alpha_{m}\right)$ is denoted by $\Gamma\left(\alpha_{1}, \ldots, \alpha_{m}\right)$. Let $\Lambda_{n}=\partial \Gamma_{n}$. Then we have that

$$
\Lambda_{n}=\bigcup_{\left(\alpha_{1}, \ldots, \alpha_{m}\right)} \Gamma\left(\alpha_{1}, \ldots, \alpha_{m}\right),
$$

where the union covers all partitions $\left(\alpha_{1}, \ldots, \alpha_{m}\right)$ of $\mathbf{n}$ for $m \geq 2$.

The permuto-associahedra $\Gamma_{2}$ and $\Gamma_{3}$ are illustrated by Figure 3 (see [29, p. 314] for the 3-dimensional permuto-associahedron $\Gamma_{4}$ ).

Here we briefly explain how to label the permuto-associahedron $\Gamma_{3}$ in Figure 3 Recall that the permuto-associahedra are used to describe the higher homotopy 

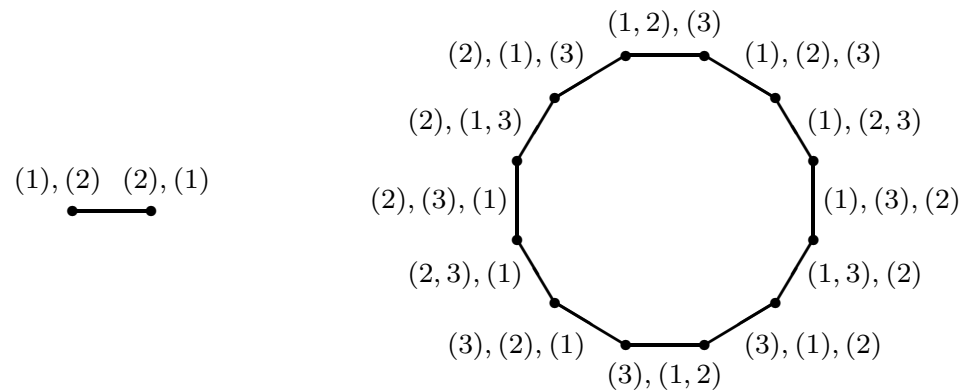

Figure 3 . The permuto-associahedra $\Gamma_{2}$ and $\Gamma_{3}$

commutativity of $A_{n}$-spaces. When the multiplication of the space is strictly associative, the permutohedra are enough to describe it. Then we expect that the permuto-associahedra $\Gamma_{n}$ are given by modifying the permutohedra $P_{n}$. In fact, $\Gamma_{n}$ is given by cutting off all the faces of $P_{n}$ in an appropriate way, so that each of the facets of $\Gamma_{n}$ corresponds to one of the faces of $P_{n}$. Let us explain the case of $n=3$. In Figure 11, the left hexagon is $P_{3}$ and the right dodecagon is $\Gamma_{3}$. In those pictures, we need to take $x, y$ and $z$ as 1,2 and 3 , respectively. The uppermost edge is a commuting homotopy between $x y$ and $y x$, and thus it is relabeled by $((1,2),(3))$. The vertex labeled by $x y z$ in the left hexagon is relabeled by the partition $((1),(2),(3))$ in the right dodecagon. Since each of the faces of $P_{3}$ gives a facet of $\Gamma_{3}$, to make $\Gamma_{3}$, the vertex $x y z$ in $P_{3}$ is replaced by an edge which is $K_{3}$ representing the associating homotopy between $(x y) z$ and $x(y z)$.

Now we construct the face operators for $\Gamma_{n}$. If $\left(\alpha_{1}, \ldots, \alpha_{m}\right)$ is a partition of $\mathbf{n}$ of type $\left(t_{1}, \ldots, t_{m}\right)$, then the facet $\Gamma\left(\alpha_{1}, \ldots, \alpha_{m}\right)$ is homeomorphic to $K_{m} \times \Gamma_{t_{1}} \times$ $\cdots \times \Gamma_{t_{m}}$ by the face operator $\epsilon^{\left(\alpha_{1}, \ldots, \alpha_{m}\right)}: K_{m} \times \Gamma_{t_{1}} \times \cdots \times \Gamma_{t_{m}} \rightarrow \Gamma\left(\alpha_{1}, \ldots, \alpha_{m}\right)$ (see [8, p. 139]).

First we give a rough idea of the construction. Again, let us explain the case of $n=3$. In the right dodecagon of Figure 3 the uppermost edge labeled with $((1,2),(3))$ is homeomorphic to $K_{2} \times \Gamma_{2} \times \Gamma_{1}$ by the face operator $\epsilon^{((1,2),(3))}$. On the other hand, the edge labeled with $((1),(2),(3))$ is homeomorphic to $K_{3} \times \Gamma_{1} \times$ $\Gamma_{1} \times \Gamma_{1}$ by $\epsilon^{((1),(2),(3))}$. The intersection of these two edges is a vertex, the images of $\left(*, \epsilon^{((1),(2))}(*, *, *), *\right)$ by $\epsilon^{((1,2),(3))}$ and $\left(\partial_{1}(2,2)(*, *), *, *, *\right)$ by $\epsilon^{((1),(2),(3))}$, where $\partial_{1}(2,2): K_{2} \times K_{2} \rightarrow K_{3}$ denotes a face operator of the associahedron $K_{3}$.

The next right vertex is the intersection of the two edges labeled with $((1),(2),(3))$ and $((1),(2,3))$, the images of $\left(\partial_{2}(2,2)(*, *), *, *, *\right)$ in $K_{3} \times \Gamma_{1} \times \Gamma_{1} \times \Gamma_{1}$ by $\epsilon^{((1),(2),(3))}$ and $\left(*, *, \epsilon^{((1),(2))}(*, *, *)\right)$ in $K_{2} \times \Gamma_{1} \times \Gamma_{2}$ by $\epsilon^{((1),(2,3))}$. Another next vertex, the intersection of the two edges $((1),(2,3))$ and $((1),(3),(2))$, is the images of $\left(*, *, \epsilon^{((2),(1))}(*, *, *)\right)$ in $K_{2} \times \Gamma_{1} \times \Gamma_{2}$ by $\epsilon^{((1),(2,3))}$ and $\left(\partial_{2}(2,2)(*, *), *, *, *\right)$ in $K_{3} \times \Gamma_{1} \times \Gamma_{1} \times \Gamma_{1}$ by $\epsilon^{((1),(3),(2))}$.

In $\Gamma_{4}$, the facet labeled with $((1,2,4),(3))$ is a dodecagon homeomorphic to $K_{2} \times \Gamma_{3} \times \Gamma_{1}$, while the facet labeled with $((1,4),(2),(3))$ is a square homeomorphic to $K_{3} \times \Gamma_{2} \times \Gamma_{1} \times \Gamma_{1}$. The intersection of these two facets is an edge which is also the images of $\left(*, \epsilon^{((1,3),(2))}(*, t, *), *\right)$ by $\epsilon^{((1,2,4),(3))}$ and $\left(\partial_{2}(2,2)(*, *), t, *\right)$ by $\epsilon^{((1,4),(2),(3))}$ for $t \in \Gamma_{2}$. 
In general, we have the following:

Proposition 2.1. Let $\left(\alpha_{1}, \ldots, \alpha_{m}\right)$ be a partition of $\mathbf{n}$ of type $\left(t_{1}, \ldots, t_{m}\right)$ with $m \geq 2$. Then we have the following relations:

(1) If $r, s \geq 2$ with $r+s=m+1$ and $1 \leq k \leq r$, then

$$
\begin{aligned}
& \epsilon^{\left(\alpha_{1}, \ldots, \alpha_{m}\right)}\left(\partial_{k}(r, s)(\rho, \sigma), \tau_{1}, \ldots, \tau_{m}\right) \\
& =\epsilon^{\left(\beta_{1}, \ldots, \beta_{r}\right)}\left(\rho, \tau_{1}, \ldots, \tau_{k-1}, \epsilon^{\left(\gamma_{1}, \ldots, \gamma_{s}\right)}\left(\sigma, \tau_{k}, \ldots, \tau_{k+s-1}\right), \tau_{k+s}, \ldots, \tau_{m}\right),
\end{aligned}
$$

where $\left(\beta_{1}, \ldots, \beta_{r}\right)$ is the partition of $\mathbf{n}$ of type $\left(t_{1}, \ldots, t_{k-1}, t_{k}+\cdots+t_{k+s-1}, t_{k+s}\right.$, $\left.\ldots, t_{m}\right)$ defined by

$$
\beta_{i}(t)= \begin{cases}\alpha_{i}(t) & \text { for } 1 \leq i \leq k-1,1 \leq t \leq t_{i} \\ \alpha_{i+s-1}(t) & \text { for } k+1 \leq i \leq r, 1 \leq t \leq t_{i+s-1}\end{cases}
$$

and

$$
\beta_{k}=\alpha_{k} \cup \cdots \cup \alpha_{k+s-1},
$$

and $\left(\gamma_{1}, \ldots, \gamma_{s}\right)$ is the partition of $\left(1, \ldots, t_{k}+\cdots+t_{k+s-1}\right)$ of type $\left(t_{k}, \ldots, t_{k+s-1}\right)$ given by $\beta_{k} \gamma_{i}(t)=\alpha_{i+k-1}(t)$ for $1 \leq i \leq s$ and $1 \leq t \leq t_{i+k-1}$.

(2) If $\left(\zeta_{1}, \ldots, \zeta_{l}\right)$ is a partition of $\left(1, \ldots, t_{k}\right)$ of type $\left(u_{1}, \ldots, u_{l}\right)$ with $l \geq 2$, then

$$
\begin{aligned}
& \epsilon^{\left(\alpha_{1}, \ldots, \alpha_{m}\right)}\left(\rho, \tau_{1}, \ldots, \tau_{k-1}, \epsilon^{\left(\zeta_{1}, \ldots, \zeta_{l}\right)}\left(\sigma, \omega_{1}, \ldots, \omega_{l}\right), \tau_{k+1}, \ldots, \tau_{m}\right) \\
& =\epsilon^{\left(\eta_{1}, \ldots, \eta_{q}\right)}\left(\partial_{k}(m, l)(\rho, \sigma), \tau_{1}, \ldots, \tau_{k-1}, \omega_{1}, \ldots, \omega_{l}, \tau_{k+1}, \ldots, \tau_{m}\right),
\end{aligned}
$$

where $q=m+l-1$ and $\left(\eta_{1}, \ldots, \eta_{q}\right)$ is the partition of $\mathbf{n}$ of type $\left(t_{1}, \ldots, t_{k-1}, u_{1}, \ldots\right.$, $\left.u_{l}, t_{k+1}, \ldots, t_{m}\right)$ defined by

$$
\eta_{i}(t)= \begin{cases}\alpha_{i}(t) & \text { for } 1 \leq i \leq k-1,1 \leq t \leq t_{i}, \\ \alpha_{k} \zeta_{i-k+1}(t) & \text { for } k \leq i \leq k+l-1,1 \leq t \leq u_{i-k+1}, \\ \alpha_{i-l+1}(t) & \text { for } k+l \leq i \leq q, 1 \leq t \leq t_{i-l+1} .\end{cases}
$$

Remark 2.2. In Proposition 2.1, the statements (1) and (2) are equivalent. In fact, the partitions $\left(\alpha_{1}, \ldots, \alpha_{m}\right),\left(\beta_{1}, \ldots, \beta_{r}\right)$ and $\left(\gamma_{1}, \ldots, \gamma_{s}\right)$ in (1) correspond to the partitions $\left(\eta_{1}, \ldots, \eta_{q}\right),\left(\alpha_{1}, \ldots, \alpha_{m}\right)$ and $\left(\zeta_{1}, \ldots, \zeta_{l}\right)$ in $(2)$, respectively.

Next we construct the degeneracy operators $\delta_{j}: \Gamma_{n} \rightarrow \Gamma_{n-1}$ for $1 \leq j \leq n$. Let $e$ be a face of $\Gamma_{n}$ represented by an insertion of parentheses of a partition $\left(\alpha_{1}, \ldots, \alpha_{m}\right)$ of $\mathbf{n}$. To get the representation of $\delta_{j}(e)$, we remove $j$ in the partition $\left(\alpha_{1}, \ldots, \alpha_{m}\right)$ and replace $k$ by $k-1$ if $k>j$. Then we modify naturally to get a parenthesized sequence in a meaningful way.

For example, if $e$ is the edge represented by $\left(\alpha_{1}, \alpha_{2}\right)$ in $\Gamma_{3}$ with $\alpha_{1}=(1,2)$ and $\alpha_{2}=(3)$, then $\delta_{2}(e)$ is the vertex of $\Gamma_{2}$ represented by $\left(\beta_{1}, \beta_{2}\right)$ with $\beta_{1}=(1)$ and $\beta_{2}=(2)$, and $\delta_{3}(e)$ is the edge represented by $(1,2)$. As another example, let $v$ be the vertex of $\Gamma_{4}$ represented by $(((1),(2)),((3),(4)))$. Then $\delta_{3}(v)$ is the vertex of $\Gamma_{3}$ represented by $(((1),(2)),(3))$.

In general, we have the following result by using a similar argument to the proof of [22, I, Prop. 3] (see also [18, Lemma 4.5]):

Proposition 2.3. Let $n \geq 1$. Then there is a collection of degeneracy operators $\left\{\delta_{j}: \Gamma_{n} \rightarrow \Gamma_{n-1}\right\}_{1 \leq j \leq n}$ satisfying the following conditions:

(1) Assume that $\left(\alpha_{1}, \ldots, \alpha_{m}\right)$ is a partition of $\mathbf{n}$ of type $\left(t_{1}, \ldots, t_{m}\right)$ with $m \geq 2$. If $1 \leq j \leq n$, then we can choose $1 \leq k \leq m$ and $1 \leq t \leq t_{k}$ with $\alpha_{k}(t)=j$. 
(i) If $t_{k} \geq 2$, then

$\delta_{j} \epsilon^{\left(\alpha_{1}, \ldots, \alpha_{m}\right)}\left(\sigma, \tau_{1}, \ldots, \tau_{m}\right)=\epsilon^{\left(\tilde{\alpha}_{1}, \ldots, \tilde{\alpha}_{m}\right)}\left(\sigma, \tau_{1}, \ldots, \tau_{k-1}, \delta_{t}\left(\tau_{k}\right), \tau_{k+1}, \ldots, \tau_{m}\right)$, where $\left(\tilde{\alpha}_{1}, \ldots, \tilde{\alpha}_{m}\right)$ is the partition of $(1, \ldots, n-1)$ of type $\left(t_{1}, \ldots, t_{k-1}, t_{k}-1, t_{k+1}\right.$, $\left.\ldots, t_{m}\right)$ given by

$$
\tilde{\alpha}_{k}(s)= \begin{cases}\alpha_{k}(s) & \text { if } \alpha_{k}(s)<j \\ \alpha_{k}(s+1)-1 & \text { if } \alpha_{k}(s) \geq j\end{cases}
$$

and for $l \neq k$,

$$
\tilde{\alpha}_{l}(s)= \begin{cases}\alpha_{l}(s) & \text { if } \alpha_{l}(s)<j, \\ \alpha_{l}(s)-1 & \text { if } \alpha_{l}(s)>j .\end{cases}
$$

(ii) If $m \geq 3$ and $t_{k}=1$, then

$$
\begin{aligned}
& \delta_{j} \epsilon^{\left(\alpha_{1}, \ldots, \alpha_{m}\right)}\left(\sigma, \tau_{1}, \ldots, \tau_{m}\right) \\
& =\epsilon^{\left(\tilde{\alpha}_{1}, \ldots, \tilde{\alpha}_{k-1}, \tilde{\alpha}_{k+1}, \ldots, \tilde{\alpha}_{m}\right)}\left(\theta_{k}(\sigma), \tau_{1}, \ldots, \tau_{k-1}, \tau_{k+1}, \ldots, \tau_{m}\right),
\end{aligned}
$$

where $\theta_{k}: K_{m} \rightarrow K_{m-1}$ denotes the degeneracy operator of the associahedron $K_{m}$ in [22, I, Prop. 3], and $\left(\tilde{\alpha}_{1}, \ldots, \tilde{\alpha}_{k-1}, \tilde{\alpha}_{k+1}, \ldots, \tilde{\alpha}_{m}\right)$ is the partition of $(1, \ldots, n-1)$ of type $\left(t_{1}, \ldots, t_{k-1}, t_{k+1}, \ldots, t_{m}\right)$ given by (2.3).

(iii) If $m=2$ and $t_{k}=1$, then

$$
\delta_{j} \epsilon^{\left(\alpha_{1}, \alpha_{2}\right)}\left(*, \tau_{1}, \tau_{2}\right)= \begin{cases}\tau_{2} & \text { for } k=1, \\ \tau_{1} & \text { for } k=2 .\end{cases}
$$

(2) If $i \leq j$, then $\delta_{j} \delta_{i}=\delta_{i} \delta_{j+1}$.

Remark 2.4. In general, $\delta_{j}: \Gamma_{n} \rightarrow \Gamma_{n-1}$ maps the two facets $((1, \ldots, j-1$, $j+1, \ldots, n),(j))$ and $((j),(1, \ldots, j-1, j+1, \ldots, n))$ homeomorphically onto $\Gamma_{n-1}$. On the other hand, each of the other facets in $\Gamma_{n}$ goes to the corresponding facet in $\Gamma_{n-1}$. The reason why the first two facets map homeomorphically onto $\Gamma_{n-1}$ is that those cases correspond to (iii) in Proposition 2.3 (1).

For example, $\delta_{1}: \Gamma_{3} \rightarrow \Gamma_{2}$ maps the two edges $((2,3),(1))$ and $((1),(2,3))$ of $\Gamma_{3}$ homeomorphically onto $\Gamma_{2}$, and each of other ten edges of $\Gamma_{3}$ corresponds to one of the vertices $((1),(2))$ and $((2),(1))$ of $\Gamma_{2}$.

Milgram [18 introduced the permutohedra $\left\{P_{n}\right\}_{n \geq 1}$ to construct approximations to the iterated loop spaces $\left\{\Omega^{n} \Sigma^{n} X\right\}_{n \geq 1}$. A few years later, Williams [26] used these complexes to define a higher homotopy commutativity of associative $H$-spaces.

Let $\mathbf{n}=(1, \ldots, n)$. Then we can regard $\mathbf{n}$ as a point of $\mathbf{R}^{n}$. The symmetric group $\Sigma_{n}$ on $n$ letters acts on $\mathbf{R}^{n}$ by the permutation of the coordinates. According to Milgram [18, Def. 4.1], the permutohedron $P_{n}$ is defined as the convex hull of the orbit of $\mathbf{n}$ under the action, and is homeomorphic to the $(n-1)$-dimensional ball for $n \geq 1$ (see also [26, Def. 2]). Here we illustrate the permutohedra $P_{2}$ and $P_{3}$ by Figure 4, Let $T_{n}=\partial P_{n}$. Then by [26, Thm. 3],

$$
T_{n}=\bigcup_{(\kappa, \nu)} P(\kappa, \nu)
$$

where the union covers all partitions $(\kappa, \nu)$ of $\mathbf{n}$ of type $(u, v)$ for $u, v \geq 1$ with $u+v=n$. By [26, Thm. 3], the facet $P(\kappa, \nu)$ is homeomorphic to $P_{u} \times P_{v}$ by the face operator $\epsilon^{(\kappa, \nu)}: P_{u} \times P_{v} \rightarrow P(\kappa, \nu)$ (see also [18, Lemma 4.2]). 

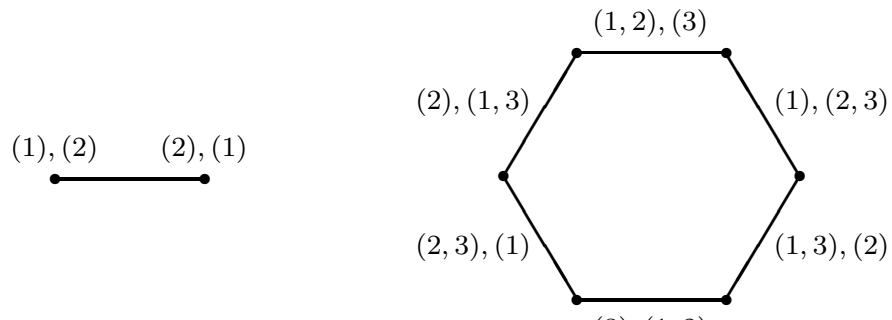

$(3),(1,2)$

Figure 4 . The permutohedra $P_{2}$ and $P_{3}$

Let $n \geq 1$. Assume that $\left(\alpha_{1}, \ldots, \alpha_{m}\right)$ is a partition of $\mathbf{n}$ of type $\left(t_{1}, \ldots, t_{m}\right)$ for $m \geq 1$ and $t_{1}, \ldots, t_{m} \geq 1$ with $t_{1}+\cdots+t_{m}=n$. Let $A\left(\alpha_{1}, \ldots, \alpha_{m}\right)$ be the complex defined by

$$
A\left(\alpha_{1}, \ldots, \alpha_{m}\right)=I \times \Gamma\left(\alpha_{1}, \ldots, \alpha_{m}\right)
$$

for $m \geq 2$, where $I$ is the unit interval and $\Gamma\left(\alpha_{1}, \ldots, \alpha_{m}\right)$ denotes the facet of $\Gamma_{n}$ corresponding to the partition $\left(\alpha_{1}, \ldots, \alpha_{m}\right)$ (see (2.1)). For $m=1$, the partition $\alpha=\mathbf{n}$, and we put $A(\mathbf{n})=\Gamma_{n}$.

In the proof of Proposition [3.4, we need the following result:

Proposition 2.5. Let $n \geq 1$. Then we have the following:

(1) The $(n-1)$-dimensional permutohedron $P_{n}$ is decomposed by

$$
P_{n}=\bigcup_{\left(\alpha_{1}, \ldots, \alpha_{m}\right)} A\left(\alpha_{1}, \ldots, \alpha_{m}\right),
$$

where the union covers all partitions $\left(\alpha_{1}, \ldots, \alpha_{m}\right)$ of $\mathbf{n}$ with $m \geq 1$.

(2) If $\left(\alpha_{1}, \ldots, \alpha_{m}\right)$ is a partition of $\mathbf{n}$ of type $\left(t_{1}, \ldots, t_{m}\right)$, then $A\left(\alpha_{1}, \ldots, \alpha_{m}\right)$ is homeomorphic to $K_{m+1} \times \Gamma_{t_{1}} \times \cdots \times \Gamma_{t_{m}}$ by the operator $\iota^{\left(\alpha_{1}, \ldots, \alpha_{m}\right)}: K_{m+1} \times \Gamma_{t_{1}} \times$ $\cdots \times \Gamma_{t_{m}} \rightarrow A\left(\alpha_{1}, \ldots, \alpha_{m}\right)$.

By using a similar way to the proof of [22, I, Prop. 25], we can show the following lemma:

Lemma 2.6. There is a collection of homeomorphisms $\left\{\zeta_{m}: I \times K_{m} \rightarrow K_{m+1}\right\}_{m \geq 2}$ satisfying the following conditions:

$$
\begin{gathered}
\zeta_{m}(0, \sigma)=\partial_{1}(2, m)(*, \sigma) . \\
\zeta_{m}\left(t, \partial_{k}(r, s+1)(\rho, \sigma)\right)=\partial_{k}(r+1, s+1)\left(\zeta_{r}(t, \rho), \sigma\right)
\end{gathered}
$$

for $r \geq 2, s \geq 1$ with $r+s=m$ and $1 \leq k \leq r$.

$$
\theta_{j} \zeta_{m}(t, \sigma)=\zeta_{m-1}\left(t, \theta_{j}(\sigma)\right)
$$

for $1 \leq j \leq m$.

Proof of Proposition 2.5. We prove by induction on $n$. Since $P_{1}=K_{2}=\Gamma_{1}=*$, it is clear for $n=1$. Now we put

$$
U_{n}=\Gamma_{n} \cup_{\{0\} \times \Lambda_{n}} I \times \Lambda_{n},
$$

where $\{0\} \times \Lambda_{n}$ is identified with $\Lambda_{n} \subset \Gamma_{n}$. Then it is clear that $U_{n}$ is homeomorphic to the $(n-1)$-dimensional ball. Now $A(\mathbf{n})=\Gamma_{n} \subset U_{n}$. Let $\iota^{(\mathbf{n})}: K_{2} \times \Gamma_{n} \rightarrow$ $A(\mathbf{n})$ denote the operator given by $\iota^{(\mathbf{n})}(*, \tau)=\tau$. If $m \geq 2$, then by Lemma 
2.6, we can identify the associahedron $K_{m+1}$ with $I \times K_{m}$ by the homeomorphism $\zeta_{m}: I \times K_{m} \rightarrow K_{m+1}$. Assume that $\left(\alpha_{1}, \ldots, \alpha_{m}\right)$ is a partition of $\mathbf{n}$ of type $\left(t_{1}, \ldots, t_{m}\right)$ with $m \geq 2$. Then $A\left(\alpha_{1}, \ldots, \alpha_{m}\right)=I \times \Gamma\left(\alpha_{1}, \ldots, \alpha_{m}\right) \subset U_{n}$. Let $\iota^{\left(\alpha_{1}, \ldots, \alpha_{m}\right)}: K_{m+1} \times \Gamma_{t_{1}} \times \cdots \times \Gamma_{t_{m}} \rightarrow A\left(\alpha_{1}, \ldots, \alpha_{m}\right)$ denote the operator given by

$$
\iota^{\left(\alpha_{1}, \ldots, \alpha_{m}\right)}\left(\zeta_{m}(t, \sigma), \tau_{1}, \ldots, \tau_{m}\right)=\left(t, \epsilon^{\left(\alpha_{1}, \ldots, \alpha_{m}\right)}\left(\sigma, \tau_{1}, \ldots, \tau_{m}\right)\right) .
$$

By (2.1) and (2.7), we see that

$$
U_{n}=\bigcup_{\left(\alpha_{1}, \ldots, \alpha_{m}\right)} A\left(\alpha_{1}, \ldots, \alpha_{m}\right)
$$

where the union covers all partitions $\left(\alpha_{1}, \ldots, \alpha_{m}\right)$ of $\mathbf{n}$ with $m \geq 1$. If we show that $U_{n}$ is the $(n-1)$-dimensional permutohedron, then we have the required conclusion.

Let $V_{n}=\partial U_{n}$. Since

$$
\zeta_{m}\left(\{1\} \times K_{m}\right)=\bigcup_{r, s} K_{r+1}(r+1, s+1),
$$

we have that

$$
V_{n}=\bigcup_{\left(\alpha_{1}, \ldots, \alpha_{m}\right)} \iota^{\left(\alpha_{1}, \ldots, \alpha_{m}\right)}\left(\left(\bigcup_{r, s} K_{r+1}(r+1, s+1)\right) \times \Gamma_{t_{1}} \times \cdots \times \Gamma_{t_{m}}\right),
$$

where $\left(\alpha_{1}, \ldots, \alpha_{m}\right)$ is a partition of $\mathbf{n}$ of type $\left(t_{1}, \ldots, t_{m}\right)$ with $m \geq 2$, and $r, s \geq 1$ with $r+s=m$.

To prove that $V_{n}$ is homeomorphic to $T_{n}=\partial P_{n}$, we need to define a collection of face operators on $V_{n}$ satisfying the conditions of [26, Thm. 3]. Assume that $(\kappa, \nu)$ is a partition of $\mathbf{n}$ of type $(u, v)$. By the inductive hypothesis, there are decompositions

$$
P_{u}=\bigcup_{\left(\eta_{1}, \ldots, \eta_{r}\right)} A\left(\eta_{1}, \ldots, \eta_{r}\right)
$$

and

$$
P_{v}=\bigcup_{\left(\lambda_{1}, \ldots, \lambda_{s}\right)} A\left(\lambda_{1}, \ldots, \lambda_{s}\right)
$$

where the unions cover all partitions $\left(\eta_{1}, \ldots, \eta_{r}\right)$ of $\mathbf{u}$ with $r \geq 1$ and $\left(\lambda_{1}, \ldots, \lambda_{s}\right)$ of $\mathbf{v}$ with $s \geq 1$, respectively. If $\left(\eta_{1}, \ldots, \eta_{r}\right)$ and $\left(\lambda_{1}, \ldots, \lambda_{s}\right)$ are of types $\left(u_{1}, \ldots, u_{r}\right)$ and $\left(v_{1}, \ldots, v_{s}\right)$, then there are homeomorphisms $\iota\left(\eta_{1}, \ldots, \eta_{r}\right): K_{r+1} \times \Gamma_{u_{1}} \times \cdots \times$ $\Gamma_{u_{r}} \rightarrow A\left(\eta_{1}, \ldots, \eta_{r}\right)$ and $\iota^{\left(\lambda_{1}, \ldots, \lambda_{s}\right)}: K_{s+1} \times \Gamma_{v_{1}} \times \cdots \times \Gamma_{v_{s}} \rightarrow A\left(\lambda_{1}, \ldots, \lambda_{s}\right)$. Put $m=r+s$. Let $\left(\alpha_{1}, \ldots, \alpha_{m}\right)$ be the partition of $\mathbf{n}$ of type $\left(u_{1}, \ldots, u_{r}, v_{1}, \ldots, v_{s}\right)$ given by

$$
\alpha_{i}(t)= \begin{cases}\kappa \eta_{i}(t) & \text { for } 1 \leq i \leq r, 1 \leq t \leq u_{i}, \\ \nu \lambda_{i-r}(t) & \text { for } r+1 \leq i \leq m, 1 \leq t \leq v_{i-r} .\end{cases}
$$

If we define a face operator $\epsilon^{(\kappa, \nu)}: P_{u} \times P_{v} \rightarrow V_{n}$ by

$$
\begin{aligned}
& \epsilon^{(\kappa, \nu)}\left(\iota^{\left(\eta_{1}, \ldots, \eta_{r}\right)}\left(\rho, \tau_{1}, \ldots, \tau_{r}\right), \iota^{\left(\lambda_{1}, \ldots, \lambda_{s}\right)}\left(\sigma, \omega_{1}, \ldots, \omega_{s}\right)\right) \\
& =\iota^{\left(\alpha_{1}, \ldots, \alpha_{m}\right)}\left(\partial_{r+1}(r+1, s+1)(\rho, \sigma), \tau_{1}, \ldots, \tau_{r}, \omega_{1}, \ldots, \omega_{s}\right),
\end{aligned}
$$

then by (2.8),

$$
V_{n}=\bigcup_{(\kappa, \nu)} \epsilon^{(\kappa, \nu)}\left(P_{u} \times P_{v}\right)
$$




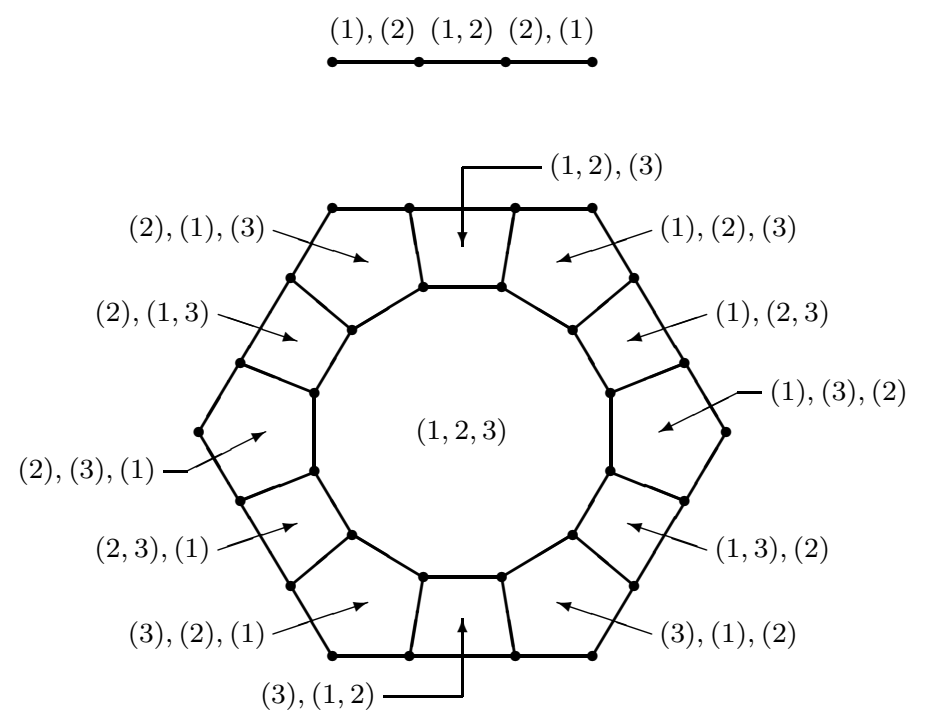

Figure 5 . The decompositions of the permutohedra $P_{2}$ and $P_{3}$

where the union covers all partitions $(\kappa, \nu)$ of $\mathbf{n}$. By using the relation

$$
\begin{aligned}
& \partial_{r+s+1}(r+s+1, t+1)\left(\partial_{r+1}(r+1, s+1) \times 1_{K_{t+1}}\right) \\
& =\partial_{r+1}(r+1, s+t+1)\left(1_{K_{r+1}} \times \partial_{s+1}(s+1, t+1)\right)
\end{aligned}
$$

for $r, s, t \geq 1$, we can show that the collection of face operators satisfies the conditions of [26, Thm. 3], and so $V_{n}$ is homeomorphic to $T_{n}=\partial P_{n}$. This implies that $U_{n}$ is the $(n-1)$-dimensional permutohedron, and we have the required conclusion. For example, the decompositions of the permutohedra $P_{2}$ and $P_{3}$ are illustrated by Figure 5. This completes the proof.

A collection of degeneracy operators $\left\{\xi_{j}: P_{n} \rightarrow P_{n-1}\right\}_{1 \leq j \leq n}$ for the permutohedra is originally constructed by Milgram [18, Lemma 4.5] (see also [26. Lemma 4]). By using Proposition 2.5 we give another construction of $\left\{\xi_{j}\right\}_{1 \leq j \leq n}$ which is useful for our arguments.

By Proposition 2.5 there is a decomposition

$$
P_{n}=\bigcup_{\left(\alpha_{1}, \ldots, \alpha_{m}\right)} A\left(\alpha_{1}, \ldots, \alpha_{m}\right),
$$

where the union covers all partitions $\left(\alpha_{1}, \ldots, \alpha_{m}\right)$ of $\mathbf{n}$ with $m \geq 1$. Let $\left(\alpha_{1}, \ldots, \alpha_{m}\right)$ be a partition of $\mathbf{n}$ of type $\left(t_{1}, \ldots, t_{m}\right)$. If $1 \leq j \leq n$, then $\alpha_{k}(t)=j$ for some $1 \leq k \leq m$ and $1 \leq t \leq t_{k}$. The map $\xi_{j}: P_{n} \rightarrow P_{n-1}$ is defined by

$$
\begin{aligned}
& \xi_{j} \iota^{\left(\alpha_{1}, \ldots, \alpha_{m}\right)}\left(\rho, \tau_{1}, \ldots, \tau_{m}\right) \\
& = \begin{cases}\iota^{\left(\tilde{\alpha}_{1}, \ldots, \tilde{\alpha}_{m}\right)}\left(\rho, \tau_{1}, \ldots, \tau_{k-1}, \delta_{t}\left(\tau_{k}\right), \tau_{k+1}, \ldots, \tau_{m}\right) & \text { if } t_{k} \geq 2, \\
\iota^{\left(\tilde{\alpha}_{1}, \ldots, \tilde{\alpha}_{k-1}, \tilde{\alpha}_{k+1}, \ldots, \tilde{\alpha}_{m}\right)}\left(\theta_{k}(\rho), \tau_{1}, \ldots, \tau_{k-1}, \tau_{k+1}, \ldots, \tau_{m}\right) & \text { if } t_{k}=1,\end{cases}
\end{aligned}
$$

where the partitions $\left(\tilde{\alpha}_{1}, \ldots, \tilde{\alpha}_{m}\right)$ and $\left(\tilde{\alpha}_{1}, \ldots, \tilde{\alpha}_{k-1}, \tilde{\alpha}_{k+1}, \ldots, \tilde{\alpha}_{m}\right)$ are defined by (2.2) -(2.3), and $\left\{\delta_{t}\right\}_{1 \leq t \leq t_{k}}$ and $\left\{\theta_{k}\right\}_{1 \leq k \leq m}$ denote the degeneracy operators for $\Gamma_{t_{k}}$ and $K_{m+1}$, respectively. Since we can show that $\left\{\xi_{j}\right\}_{1 \leq j \leq n}$ satisfies the conditions 
of [18, Lemma 4.5] (see also [26, Lemma 4]), $\left\{\xi_{j}\right\}_{1 \leq j \leq n}$ is a collection of degeneracy operators for the permutohedra $\left\{P_{n}\right\}_{n \geq 1}$.

\section{Proofs of Theorem A And Theorem B}

Stasheff [22] introduced the notion of the higher homotopy associativity of $H$ spaces. He used the associahedra $\left\{K_{i}\right\}_{2 \leq i \leq n}$ to define an $A_{n}$-form on an $H$-space. Let $n \geq 2$ and $X$ be an $H$-space with a multiplication $\mu: X \times X \rightarrow X$ such that $\mu(x, *)=\mu(*, x)=x$ for $x \in X$. An $A_{n}$-form on $X$ is a collection of maps $\left\{M_{i}: K_{i} \times X^{i} \rightarrow X\right\}_{2 \leq i \leq n}$ satisfying the following conditions:

$$
\begin{gathered}
M_{2}(*, x, y)=\mu(x, y) . \\
M_{i}\left(\partial_{k}(r, s)(\rho, \sigma), x_{1}, \ldots, x_{i}\right) \\
=M_{r}\left(\rho, x_{1}, \ldots, x_{k-1}, M_{s}\left(\sigma, x_{k}, \ldots, x_{k+s-1}\right), x_{k+s}, \ldots, x_{i}\right),
\end{gathered}
$$

where $r, s \geq 2$ with $r+s=i+1$ and $1 \leq k \leq r$.

$$
\begin{aligned}
& M_{i}\left(\tau, x_{1}, \ldots, x_{j-1}, *, x_{j+1}, \ldots, x_{i}\right) \\
& =M_{i-1}\left(\theta_{j}(\tau), x_{1}, \ldots, x_{j-1}, x_{j+1}, \ldots, x_{i}\right),
\end{aligned}
$$

where $\left\{\theta_{j}: K_{i} \rightarrow K_{i-1}\right\}_{1 \leq j \leq i}$ are the degeneracy operators. For convenience, we define that an $A_{1}$-space is just a space. For $n \geq 2$, an $A_{n}$-space is an $H$-space $X$ with a specified $A_{n}$-form on $X$. If $X$ has a collection of maps $\left\{M_{i}: K_{i} \times X^{i} \rightarrow X\right\}_{i \geq 2}$ such that $\left\{M_{i}\right\}_{2<i<n}$ is an $A_{n}$-form on $X$ for any $n \geq 2$, then $X$ is called an $A_{\infty^{-}}$ space. From the definition of an $A_{n}$-form, we see that an $A_{2}$-space and an $A_{3}$-space are an $H$-space and a homotopy associative $H$-space, respectively. Furthermore, an $A_{\infty}$-space has the homotopy type of an associative $H$-space.

Now we introduce the higher homotopy commutativity of $H$-spaces. An $A C_{n^{-}}$ form on an $A_{n}$-space is defined by using a collection of the permuto-associahedra $\left\{\Gamma_{i}\right\}_{1 \leq i \leq n}$.

Definition 3.1. Let $X$ be an $A_{n^{-}}$space with the $A_{n^{-}}$form $\left\{M_{i}\right\}_{2 \leq i \leq n}$. An $A C_{n^{-}}$ form on $X$ consists of a collection of maps $\left\{Q_{i}: \Gamma_{i} \times X^{i} \rightarrow X\right\}_{1 \leq i \leq n}$ satisfying the following conditions:

$$
\begin{gathered}
Q_{1}(*, x)=x . \\
Q_{i}\left(\epsilon^{\left(\alpha_{1}, \ldots, \alpha_{m}\right)}\left(\sigma, \tau_{1}, \ldots, \tau_{m}\right), x_{1}, \ldots, x_{i}\right) \\
=M_{m}\left(\sigma, Q_{t_{1}}\left(\tau_{1}, x_{\alpha_{1}(1)}, \ldots, x_{\alpha_{1}\left(t_{1}\right)}\right), \ldots, Q_{t_{m}}\left(\tau_{m}, x_{\alpha_{m}(1)}, \ldots, x_{\alpha_{m}\left(t_{m}\right)}\right)\right),
\end{gathered}
$$

where $\left(\alpha_{1}, \ldots, \alpha_{m}\right)$ is a partition of $\mathbf{i}$ of type $\left(t_{1}, \ldots, t_{m}\right)$.

$$
\begin{aligned}
& Q_{i}\left(\tau, x_{1}, \ldots, x_{j-1}, *, x_{j+1}, \ldots, x_{i}\right) \\
& =Q_{i-1}\left(\delta_{j}(\tau), x_{1}, \ldots, x_{j-1}, x_{j+1}, \ldots, x_{i}\right),
\end{aligned}
$$

where $\left\{\delta_{j}: \Gamma_{i} \rightarrow \Gamma_{i-1}\right\}_{1 \leq j \leq i}$ are the degeneracy operators.

An $A_{n}$-space with a specified $A C_{n}$-form is called an $A C_{n}$-space. If $X$ has a collection of maps $\left\{Q_{i}: \Gamma_{i} \times X^{i} \rightarrow X\right\}_{i \geq 1}$ such that $\left\{Q_{i}\right\}_{1 \leq i \leq n}$ is an $A C_{n}$-form on $X$ for any $n \geq 1$, then $X$ is called an $A C_{\infty}$-space.

Example 3.2. (1) $X$ is an $A C_{2}$-space if and only if $X$ is a homotopy commutative $H$-space since $Q_{2}\left(\epsilon^{((1),(2))}(*), x, y\right)=x y$ and $Q_{2}\left(\epsilon^{((2),(1))}(*), x, y\right)=y x$ for $x, y \in X$.

(2) If $X$ is an associative and commutative $H$-space, then the collection $\left\{Q_{i}\right.$ : $\left.\Gamma_{i} \times X^{i} \rightarrow X\right\}_{i \geq 1}$ defined by $Q_{i}\left(\tau, x_{1}, \ldots, x_{i}\right)=x_{1} \ldots x_{i}$ for $i \geq 1$ makes $X$ an 
$A C_{\infty}$-space. In particular, Eilenberg-Mac Lane spaces are $A C_{\infty}$-spaces by Stasheff [23, Cor. 13.10].

(3) If $X$ is an $H$-space, then by Corollary [3.6 and [26, Cor. 26], $\Omega X$ is an $A C_{\infty}$-space.

Now we recall the definition of a quasi $C_{n}$-form on an $A_{n}$-space introduced by Hemmi [5, Def. 2.1]. Let $X$ be an $A_{n}$-space and $\left\{P_{i}(X)\right\}_{1 \leq i \leq n}$ be the projective spaces of $X$. From the construction of $P_{i}(X)$, we have the inclusion $\iota_{i-1}$ : $P_{i-1}(X) \rightarrow P_{i}(X)$ and the projection $\rho_{i}: P_{i}(X) \rightarrow P_{i}(X) / P_{i-1}(X) \simeq(\Sigma X)^{(i)}$, where $(\Sigma X)^{(i)}$ denotes the $i$-fold smash product of $\Sigma X$ for $1 \leq i \leq n$. Let $J_{i}(\Sigma X)$ denote the $i$-th James reduced product space of $\Sigma X$ and $\pi_{i}: J_{i}(\Sigma X) \rightarrow(\Sigma X)^{(i)}$ be the obvious projection for $1 \leq i \leq n$. A quasi $C_{n}$-form on $X$ is a collection of maps $\left\{\psi_{i}: J_{i}(\Sigma X) \rightarrow P_{i}(X)\right\}_{1 \leq i \leq n}$ satisfying the following conditions:

$$
\begin{array}{ll}
\psi_{1}=1_{\Sigma X}: \Sigma X \longrightarrow \Sigma X . & \\
\left.\psi_{i}\right|_{J_{i-1}(\Sigma X)}=\iota_{i-1} \psi_{i-1} & \text { for } 2 \leq i \leq n . \\
\rho_{i} \psi_{i} \simeq\left(\sum_{\sigma \in \Sigma_{i}} \sigma\right) \pi_{i} & \text { for } 1 \leq i \leq n,
\end{array}
$$

where the action of the symmetric group $\Sigma_{i}$ on $(\Sigma X)^{(i)}$ is given by the permutation of the coordinates, and the summation on the right hand side is defined by using the obvious co- $H$-structure on $(\Sigma X)^{(i)}$. An $A_{n}$-space with a specified quasi $C_{n}$-form is called a quasi $C_{n}$-space. Hemmi [5, Thm. 1.1] has shown that a simply connected finite quasi $C_{p}$-space is contractible. Furthermore, Kawamoto [1] generalized the result to the case of quasi $C_{p}$-spaces with finitely generated $\bmod p$ cohomology.

Theorem 3.3 (11, Thm. B]). If $X$ is a simply connected quasi $C_{p}$-space such that the $\bmod p$ cohomology $H^{*}(X ; \mathbb{Z} / p)$ is finitely generated as an algebra, then $X$ is $\bmod p$ homotopy equivalent to a finite product of $K(\mathbb{Z}, 2) s$.

To prove Theorem A, we define a $C_{n}$-form of a map from a space to a loop space by using the permutohedra $\left\{P_{i}\right\}_{1 \leq i \leq n}$.

Let $X$ and $Y$ be spaces and $\phi: X \rightarrow \Omega Y$ be a map. A $C_{n}$-form on $\phi$ is a collection of maps $\left\{R_{i}: P_{i} \times X^{i} \rightarrow \Omega Y\right\}_{1 \leq i \leq n}$ satisfying the following conditions:

$$
\begin{gathered}
R_{1}(*, x)=\phi(x) . \\
R_{i}\left(\epsilon^{(\alpha, \beta)}(\rho, \sigma), x_{1}, \ldots, x_{i}\right) \\
=R_{r}\left(\rho, x_{\alpha(1)}, \ldots, x_{\alpha(r)}\right) \cdot R_{s}\left(\sigma, x_{\beta(1)}, \ldots, x_{\beta(s)}\right),
\end{gathered}
$$

where $(\alpha, \beta)$ is a partition of $\mathbf{i}$ of type $(r, s)$.

$$
\begin{aligned}
& R_{i}\left(\tau, x_{1}, \ldots, x_{j-1}, *, x_{j+1}, \ldots, x_{i}\right) \\
& =R_{i-1}\left(\xi_{j}(\tau), x_{1}, \ldots, x_{j-1}, x_{j+1}, \ldots, x_{i}\right),
\end{aligned}
$$

where $\left\{\xi_{j}: P_{i} \rightarrow P_{i-1}\right\}_{1 \leq j \leq i}$ are the degeneracy operators.

Now we prove the following result:

Proposition 3.4. Let $X$ be an $A_{n}$-space and $\phi_{n}: X \rightarrow \Omega P_{n}(X)$ denote the adjoint of the inclusion $\iota_{n-1} \ldots \iota_{1}: \Sigma X \rightarrow P_{n}(X)$. If $X$ admits an $A C_{n}$-form, then there is a $C_{n}$-form $\left\{R_{i}: P_{i} \times X^{i} \rightarrow \Omega P_{n}(X)\right\}_{1 \leq i \leq n}$ on $\phi_{n}$. 


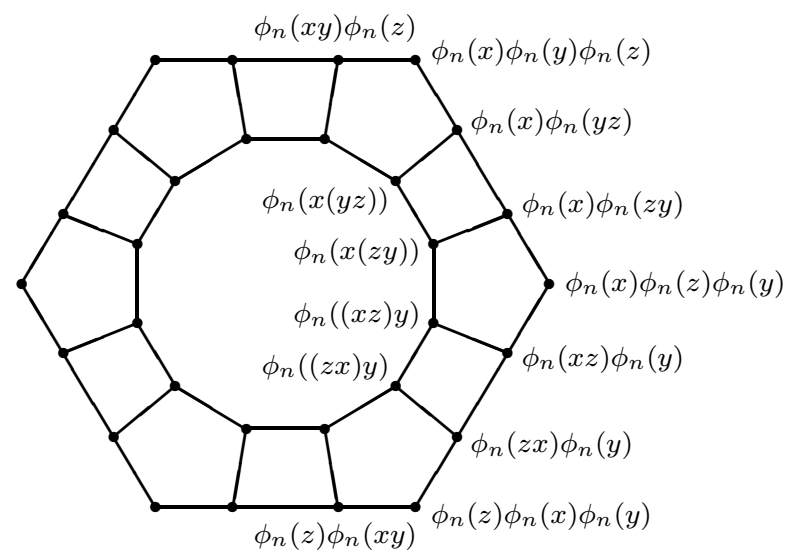

Figure 6 . The $C_{n}$-forms on $\phi_{n}$ for $n=2,3$

Proof. We prove by induction on $n$. For $n=1$, it is clear by (3.10). Suppose that we have a $C_{n-1}$-form $\left\{\tilde{R}_{i}\right\}_{1 \leq i \leq n-1}$ on $\phi_{n-1}$. If we put that $R_{i}=\iota_{n-1} \tilde{R}_{i}$ for $1 \leq i \leq n-1$, then the collection $\left\{R_{i}\right\}_{1 \leq i \leq n-1}$ is a $C_{n-1}$-form on $\phi_{n}$. According to Stasheff [23, Thm. 11.10], $\phi_{n}: X \rightarrow \Omega P_{n}(X)$ is an $A_{n}$-map, and so there is a collection of maps $\left\{F_{i}: K_{i+1} \times X^{i} \rightarrow \Omega P_{n}(X)\right\}_{1 \leq i \leq n}$ satisfying the following conditions:

$$
F_{1}(*, x)=\phi_{n}(x) .
$$

$$
\begin{aligned}
& F_{i}\left(\partial_{k}(r+1, s+1)(\rho, \sigma), x_{1}, \ldots, x_{i}\right) \\
& = \begin{cases}F_{r}\left(\rho, x_{1}, \ldots, x_{k-1}, M_{s+1}\left(\sigma, x_{k}, \ldots, x_{k+s}\right), x_{k+s+1}, \ldots, x_{i}\right) & \text { if } 1 \leq k \leq r, \\
F_{r}\left(\rho, x_{1}, \ldots, x_{r}\right) \cdot F_{s}\left(\sigma, x_{r+1}, \ldots, x_{i}\right) & \text { if } k=r+1,\end{cases}
\end{aligned}
$$

where $r, s \geq 1$ with $r+s=i$ and $1 \leq k \leq r+1$.

$$
\begin{aligned}
& F_{i}\left(\tau, x_{1}, \ldots, x_{j-1}, *, x_{j+1}, \ldots, x_{i}\right) \\
& =F_{i-1}\left(\theta_{j}(\tau), x_{1}, \ldots, x_{j-1}, x_{j+1}, \ldots, x_{i}\right)
\end{aligned}
$$

for $1 \leq j \leq i$.

By Proposition 2.5 there is a decomposition

$$
P_{n}=\bigcup_{\left(\alpha_{1}, \ldots, \alpha_{m}\right)} A\left(\alpha_{1}, \ldots, \alpha_{m}\right)
$$

where the union covers all partitions $\left(\alpha_{1}, \ldots, \alpha_{m}\right)$ of $\mathbf{n}$ with $m \geq 1$. If we define a map $R_{n}: P_{n} \times X^{n} \rightarrow \Omega P_{n}(X)$ by

$$
\begin{aligned}
& R_{n}\left(\iota^{\left(\alpha_{1}, \ldots, \alpha_{m}\right)}\left(\sigma, \tau_{1}, \ldots, \tau_{m}\right), x_{1}, \ldots, x_{n}\right) \\
& =F_{m}\left(\sigma, Q_{t_{1}}\left(\tau_{1}, x_{\alpha_{1}(1)}, \ldots, x_{\alpha_{1}\left(t_{1}\right)}\right), \ldots, Q_{t_{m}}\left(\tau_{m}, x_{\alpha_{m}(1)}, \ldots, x_{\alpha_{m}\left(t_{m}\right)}\right)\right),
\end{aligned}
$$

then the collection $\left\{R_{i}\right\}_{1 \leq i \leq n}$ satisfies the conditions (3.10)-(3.12). For example, the $C_{n}$-forms on $\phi_{n}$ for $n=2,3$ are illustrated by Figure 6 . This completes the proof. 
Now we proceed to the proof of Theorem A.

Proof of Theorem A. First we prove (1) by induction on $n$. For $n=1$, it is clear by (3.7). Suppose that we have a quasi $C_{n-1}$-form $\left\{\psi_{i}\right\}_{1<i<n-1}$ on $X$. By Proposition 3.4, there is a $C_{n}$-form $\left\{R_{i}\right\}_{1 \leq i \leq n}$ on $\phi_{n}$. For the same reason as in [28], we can assume without loss of generality that the image of $R_{i}$ is contained in the set of loops of length $i$ for $1 \leq i \leq n$. Let $\zeta_{i}:[0, i] \times P_{i} \times X^{i} \rightarrow P_{n}(X)$ be the adjoint of $R_{i}$ for $1 \leq i \leq n$. It is shown by Williams [27] that there is a map $\tau_{i}:[0, i] \times P_{i} \rightarrow I^{i}$ satisfying suitable conditions (see also Milgram [18, Lemma 4.6]). Let $\kappa_{i}:[0, i] \times P_{i} \times X^{i} \rightarrow J_{i}(\Sigma X)$ be the map defined by $\kappa_{i}=\chi_{i}\left(\tau_{i} \times 1_{X^{i}}\right)$, where $\chi_{i}: I^{i} \times X^{i} \rightarrow J_{i}(\Sigma X)$ denotes the obvious projection for $1 \leq i \leq n$. Then by [28, Thm. 1.1], we have a map $\psi_{n}: J_{n}(\Sigma X) \rightarrow P_{n}(X)$ which satisfies (3.8) and $\psi_{n} \kappa_{n}=\zeta_{n}$.

Here we explain the construction of $\psi_{n}$ briefly since [28, Thm. 1.1] omitted the proof. By the inductive hypothesis, we can assume that $\psi_{i} \kappa_{i}=\zeta_{i}$ for $1 \leq i \leq n-1$. It is known that $J_{n}(\Sigma X)=J_{n-1}(\Sigma X) \cup_{\eta_{n}} I^{n} \times X^{n}$, where $\eta_{n}: \partial I^{n} \times X^{n} \cup I^{n} \times$ $X^{[n]} \rightarrow J_{n-1}(\Sigma X)$ is the map defined by

$$
\eta_{n}\left(t_{1}, \ldots, t_{n}, x_{1}, \ldots, x_{n}\right)=\left(\left(t_{1}, x_{1}\right), \ldots,\left(t_{i-1}, x_{i-1}\right),\left(t_{i+1}, x_{i+1}\right), \ldots,\left(t_{n}, x_{n}\right)\right)
$$

if $t_{i} \in \partial I$ or $x_{i}=*$ for $1 \leq i \leq n$, and $X^{[n]}$ denotes the $n$-fold fat wedge of $X$ given by

$$
X^{[n]}=\left\{\left(x_{1}, \ldots, x_{n}\right) \in X^{n} \mid x_{i}=* \text { for some } 1 \leq i \leq n\right\} .
$$

Let $T_{n}=[0, n] \times P_{n} \times X^{n}$ and $S_{n}=\partial\left([0, n] \times P_{n}\right) \times X^{n} \cup[0, n] \times P_{n} \times X^{[n]}$. If we define a map $\lambda_{n}: S_{n} \rightarrow J_{n-1}(\Sigma X)$ by $\lambda_{n}=\left.\eta_{n}\left(\tau_{n} \times 1_{X^{n}}\right)\right|_{S_{n}}$, then $\iota_{n-1} \psi_{n-1} \lambda_{n}=\left.\zeta_{n}\right|_{S_{n}}$, and so there is a map $\theta_{n}: J_{n-1}(\Sigma X) \cup_{\lambda_{n}} T_{n} \rightarrow P_{n}(X)$ with $\left.\theta_{n}\right|_{J_{n-1}(\Sigma X)}=\iota_{n-1} \psi_{n-1}$ and $\left.\theta_{n}\right|_{T_{n}}=\zeta_{n}$. Since there is a homotopy equivalence $\nu_{n}: J_{n-1}(\Sigma X) \cup_{\lambda_{n}} T_{n} \rightarrow$ $J_{n}(\Sigma X)$ with $\left.\nu_{n}\right|_{J_{n-1}(\Sigma X)}=\epsilon_{n-1}$ and $\left.\nu_{n}\right|_{T_{n}}=\kappa_{n}$, we have a map $\psi_{n}: J_{n}(\Sigma X) \rightarrow$ $P_{n}(X)$ such that $\left.\psi_{n}\right|_{J_{n-1}(\Sigma X)}=\iota_{n-1} \psi_{n-1}$ and $\psi_{n} \kappa_{n} \simeq \zeta_{n}$ rel $S_{n}$. By replacing $\zeta_{n}$ with $\tilde{\zeta}_{n}=\psi_{n} \kappa_{n}$, we have the required conclusion.

Now we consider the condition (3.9). It is sufficient to show that

$$
\rho_{n} \zeta_{n} \simeq\left(\sum_{\sigma \in \Sigma_{n}} \sigma\right) \pi_{n} \kappa_{n}
$$

since $\zeta_{n}=\psi_{n} \kappa_{n}$. From the proof of Proposition 3.4, the map $R_{n}: P_{n} \times X^{n} \rightarrow$ $\Omega P_{n}(X)$ is constructed by using the $A C_{n}$-form $\left\{Q_{i}\right\}_{1 \leq i \leq n}$ on $X$ and the $A_{n}$-form $\left\{F_{i}\right\}_{1 \leq i \leq n}$ on $\phi_{n}$. Since the image of $F_{i}$ is contained in $\Omega P_{n-1}(X)$ for $1 \leq i \leq n-1$ from the proof of [23, Thm. 11.10], we have that

$$
\left(\Omega \rho_{n}\right) R_{n} \simeq\left(\bigvee_{\sigma \in \Sigma_{n}} G_{\sigma}\right) H_{n} .
$$

Here $G_{\sigma}: \Sigma^{n-1} X^{(n)} \rightarrow \Omega P_{n}(X)$ is represented by the map $\tilde{G}_{\sigma}: P_{n} \times X^{n} \rightarrow \Omega P_{n}(X)$ given by $\tilde{G}_{\sigma}\left(\tau, x_{1}, \ldots, x_{n}\right)=F_{n}\left(\tau, x_{\sigma(1)}, \ldots, x_{\sigma(n)}\right)$ for $\sigma \in \Sigma_{n}$, and

$$
H_{n}: P_{n} \times X^{n} \rightarrow \bigvee_{\sigma \in \Sigma_{n}} \Sigma^{n-1} X^{(n)}
$$

denotes the appropriate collapsing map. From these observations, we have the condition (3.9). 
Next let us prove (2). For $n=1$, it is clear by (3.4). By the inductive hypothesis, we assume that there is an $A C_{n-1}$-form $\left\{Q_{i}\right\}_{1 \leq i \leq n-1}$ on $X$.

Now we use a result of Williams [27] on the relation between higher homotopy commutativity and extension of maps. Let $g:(\Sigma X)^{\vee n} \rightarrow P_{n}(X)$ be the map defined by $\left.g\right|_{\Sigma X}=\iota_{n-1} \ldots \iota_{1}$ for each factor, where $(\Sigma X)^{\vee n}$ denotes the $n$-fold wedge of $\Sigma X$. Since $X$ is a quasi $C_{n}$-space, $g$ is extended to a map $\tilde{g}:(\Sigma X)^{n} \rightarrow$ $P_{n}(X)$ by using $\psi_{n}$. Then by [27, Thm. 2], there exists a $C_{n}$-form $\left\{R_{i}\right\}_{1 \leq i \leq n}$ on $\phi_{n}$. Now we can assume without loss of generality that the $C_{n-1}$-form $\left\{R_{i}\right\}_{1<i<n-1}$ is obtained from $\left\{Q_{i}\right\}_{1 \leq i \leq n-1}$ by using the way in the proof of Proposition 3.4 Since $\phi_{n}$ is an $A_{n}$-map, we have a map $\tilde{Q}_{n}: \Gamma_{n} \times X^{n} \rightarrow \Omega P_{n}(X)$ such that the $\left\{\tilde{Q}_{i}\right\}_{1 \leq i \leq n}$ satisfy the conditions (3.5)- (3.6), where $\tilde{Q}_{i}=\phi_{n} Q_{i}$ for $1 \leq i \leq n-1$. Since $X$ is an $A_{n+1}$-space, there is a map $\omega_{n}: \Omega P_{n}(X) \rightarrow X$ with $\omega_{n} \phi_{n}=1_{X}$. Put $Q_{n}=\omega_{n} \tilde{Q}_{n}: \Gamma_{n} \times X^{n} \rightarrow X$. Then $\left\{Q_{i}\right\}_{1 \leq i \leq n}$ is an $A C_{n}$-form on $X$, and so we have the required conclusion. This completes the proof of Theorem A.

Remark 3.5. The decompositions of the permutohedra in Proposition 2.5 play important roles in the proof of Theorem A. We note that Hemmi [5] gave another decomposition of $P_{n}$ by using the permutohedra $\left\{P_{i}\right\}_{1 \leq i \leq n-1}$ and the simplices $\left\{\Delta^{i}\right\}_{1 \leq i \leq n-1}$. He used the result to show that if $X$ is an associative $H$-space, then the quasi $C_{n}$-form on $X$ is equivalent to a $C_{n}$-form in the sense of Williams [26]. Def. 5] (see [5] Thm. 2.2]). The proof of Theorem A is regarded as a generalization of the one of [5, Thm. 2.2] to the case of $A_{n}$-spaces.

From Theorem A and the result by Hemmi [5, Thm. 2.2], we have the following result:

Corollary 3.6. Let $X$ be an associative $H$-space. Then $X$ is an $A C_{n}$-space if and only if $X$ is a $C_{n}$-space in the sense of Williams.

It is natural to consider the notion of maps between $A C_{n}$-spaces preserving $A C_{n}$-forms.

Let $X$ and $Y$ be $A_{n}$-spaces. According to Stasheff [22, II, Def. 4.1], a map $\phi: X \rightarrow Y$ is called an $A_{n}$-homomorphism if $\phi M_{i}^{X}=M_{i}^{Y}\left(1_{K_{i}} \times \phi^{i}\right)$ for $2 \leq i \leq n$, where $\left\{M_{i}^{X}\right\}_{2 \leq i \leq n}$ and $\left\{M_{i}^{Y}\right\}_{2 \leq i \leq n}$ are $A_{n}$-forms on $X$ and $Y$, respectively.

Definition 3.7. Let $X$ and $Y$ be $A C_{n}$-spaces with the $A C_{n}$-forms $\left\{Q_{i}^{X}\right\}_{1 \leq i \leq n}$ and $\left\{Q_{i}^{Y}\right\}_{1 \leq i \leq n}$, respectively. An $A_{n}$-homomorphism $\phi: X \rightarrow Y$ is called an $A C_{n}$-homomorphism if $\phi Q_{i}^{X}=Q_{i}^{Y}\left(1_{\Gamma_{i}} \times \phi^{i}\right)$ for $1 \leq i \leq n$.

Now we consider the odd dimensional sphere $\left(S^{2 n-1}\right)_{p}^{\wedge}$ completed at $p$ for $n \geq$ 1. Let $\epsilon_{n}:\left(S^{2 n-1}\right)_{p}^{\wedge} \rightarrow \Omega^{2}\left(S^{2 n+1}\right)_{p}^{\wedge}$ denote the double suspension which is the double adjoint of the identity $1_{\left(S^{2 n+1}\right)_{p}}$ on $\left(S^{2 n+1}\right)_{p}^{\wedge} \simeq \Sigma^{2}\left(S^{2 n-1}\right)_{p}^{\wedge}$. By Example $3.2(3), \Omega^{2}\left(S^{2 n+1}\right)_{p}^{\wedge}$ is an $A C_{\infty}$-space. According to Stasheff [22 I, Thm. 17], $\left(S^{2 n-1}\right)_{p}^{\wedge}$ admits an $A_{p-1}$-form so that $\epsilon_{n}:\left(S^{2 n-1}\right)_{p}^{\wedge} \rightarrow \Omega^{2}\left(S^{2 n+1}\right)_{p}^{\wedge}$ is an $A_{p-1^{-}}$ homomorphism. By using a similar argument to the proof of [22, I, Thm. 17], we can prove the following result:

Proposition 3.8. Let $p$ be a prime. Then $\left(S^{2 n-1}\right)_{p}^{\wedge}$ admits an $A C_{p-1}$-form so that the double suspension $\epsilon_{n}:\left(S^{2 n-1}\right)_{p}^{\wedge} \rightarrow \Omega^{2}\left(S^{2 n+1}\right)_{p}^{\wedge}$ is an $A C_{p-1}$-homomorphism for $n \geq 1$. 
In the proof of Theorem B, we need the following lemma:

Lemma 3.9. If $X$ is a connected $A C_{n}$-space, then the universal covering $\tilde{X}$ is a simply connected $A C_{n}$-space and the covering projection map $\omega: \tilde{X} \rightarrow X$ is an $A C_{n}$-homomorphism.

Proof. We give an outline of the proof. Let $\left\{M_{i}\right\}_{2 \leq i \leq n}$ and $\left\{Q_{i}\right\}_{1 \leq i \leq n}$ be the $A_{n^{-}}$ form and the $A C_{n}$-form on $X$, respectively. From the covering lifting property (cf. 19, Ch. 2, Lemma 1.7]), there are maps $\tilde{M}_{i}: K_{i} \times \tilde{X}^{i} \rightarrow \tilde{X}$ and $\tilde{Q}_{i}: \Gamma_{i} \times \tilde{X}^{i} \rightarrow \tilde{X}$ such that $\omega \tilde{M}_{i}=M_{i}\left(1_{K_{i}} \times \omega^{i}\right)$ for $2 \leq i \leq n$ and $\omega \tilde{Q}_{i}=Q_{i}\left(1_{\Gamma_{i}} \times \omega^{i}\right)$ for $1 \leq i \leq$ $n$. From the uniqueness of the lifting, the collections $\left\{\tilde{M}_{i}\right\}_{2 \leq i \leq n}$ and $\left\{\tilde{Q}_{i}\right\}_{1 \leq i \leq n}$ satisfy the conditions (3.1)-3.3) and (3.4)-3.6), respectively. This completes the proof.

Now we proceed to the proof of Theorem B.

Proof of Theorem $B$. Let $X$ be a connected $A C_{p}$-space with finitely generated $\bmod p$ cohomology. If $\tilde{X}$ denotes the universal covering of $X$, then there is an $H$-fibration

$$
\tilde{X} \longrightarrow X \longrightarrow K\left(\pi_{1}(X), 1\right),
$$

where $K\left(\pi_{1}(X), 1\right)$ has the $\bmod p$ homotopy type of a finite product of $K(\mathbb{Z}, 1) \mathrm{s}$ and $K\left(\mathbb{Z} / p^{i}, 1\right)$ s for $i \geq 1$. According to Browder [4, there is a version of the Serre spectral sequence associated to the $H$-fibration (3.14). As in the argument of [7, §3], we see that the $\bmod p$ cohomology $H^{*}(\tilde{X} ; \mathbb{Z} / p)$ is finitely generated as an algebra. From Theorem A, Theorem 3.3 and Lemma 3.9, $\tilde{X}$ is $\bmod p$ homotopy equivalent to a finite product of $K(\mathbb{Z}, 2)$ s. For dimensional reasons, the spectral sequence associated to the $H$-fibration (3.14) collapses. Hence we have that

$$
H^{*}(X ; \mathbb{Z} / p) \cong H^{*}\left(K\left(\pi_{1}(X), 1\right) ; \mathbb{Z} / p\right) \otimes H^{*}(\tilde{X} ; \mathbb{Z} / p),
$$

and there is a map $\zeta: X \rightarrow K\left(\pi_{1}(X), 1\right) \times \tilde{X}$ which induces an isomorphism on the $\bmod p$ cohomology. Then $\zeta$ is a $\bmod p$ homotopy equivalence (cf. [19, Ch. 4 , Cor. 1.6]), and so we have the required conclusion. This completes the proof of Theorem B.

\section{REFERENCES}

[1] J. Aguadé and L. Smith, On the mod p torus theorem of John Hubbuck, Math. Z. 191 (1986), 325-326. MR 87e:57044

[2] A. Bousfield and D. Kan, Homotopy limits, completions and localizations, Lecture Notes in Math. 304, Springer-Verlag, 1972. MR 51:1825

[3] C. Broto and J. A. Crespo, H-spaces with noetherian mod two cohomology algebra, Topology 38 (1999), 353-386. MR 99i:55013

[4] W. Browder, The cohomology of covering spaces of $H$-spaces, Bull. Amer. Math. Soc. 65 (1959), 140-141. MR 22:1891

[5] Y. Hemmi, Higher homotopy commutativity of $H$-spaces and the $\bmod p$ torus theorem, Pacific J. Math. 149 (1991), 95-111. MR 92a:55010

[6] J. R. Hubbuck, On homotopy commutative H-spaces, Topology 8 (1969), 119-126. MR 38:6592

[7] R. M. Kane, The homology of Hopf spaces, North-Holland Math. Library 40, North-Holland, 1988. MR 90f:55018

[8] M. M. Kapranov, The permutoassociahedron, Mac Lane's coherence theorem and asymptotic zones for the KZ equation, J. Pure Appl. Algebra 85 (1993), 119-142. MR 94b:52017

[9] Y. Kawamoto, Loop spaces of $H$-spaces with finitely generated cohomology, Pacific J. Math. 190 (1999), 311-328. MR 2000i:55027 
[10] - Homotopy classification of higher homotopy commutative loop spaces with finitely generated cohomology, Hiroshima Math. J. 30 (2000), 317-344. MR 2001e:55011

[11] - Higher homotopy commutativity of H-spaces with finitely generated cohomology, Pacific J. Math. 204 (2002), 145-161. MR 2003d:55010

[12] Y. Kawamoto and J. P. Lin, Homotopy commutativity of $H$-spaces with finitely generated cohomology, Trans. Amer. Math. Soc. 353 (2001), 4481-4496. MR 2002g:55017

[13] J. P. Lin, A cohomological proof of the torus theorem, Math. Z. 190 (1985), 469-476. MR 87c:55009

[14] Loops of $H$-spaces with finitely generated cohomology rings, Topology Appl. 60 (1994), 131-152. MR 95m:55022

[15] — H-spaces with finiteness conditions, Handbook of Algebraic Topology, edited by I.M. James, North-Holland, 1995, 1095-1141. MR 97c:55017

[16] J. P. Lin and F. D. Williams, Homotopy-commutative $H$-spaces, Proc. Amer. Math. Soc. 113 (1991), 857-865. MR 92b:55011

[17] C. A. McGibbon, Higher forms of homotopy commutativity and finite loop spaces, Math. Z. 201 (1989), 363-374. MR 90f:55019

[18] R. J. Milgram, Iterated loop spaces, Ann. of Math. 84 (1966), 386-403. MR 34:6767

[19] M. Mimura and H. Toda, Topology of Lie groups, I and II, Trans. Math. Monographs 91, Amer. Math. Soc., 1991. MR 92h:55001

[20] V. Reiner and G. M. Ziegler, Coxeter-associahedra, Mathematika 41 (1994), 364-393. MR 95m:52023

[21] M. Slack, A classification theorem for homotopy commutative mod $2 \mathrm{H}$-spaces with finitely generated cohomology rings, Mem. Amer. Math. Soc. 92 (1991). MR 92k:55015

[22] J. D. Stasheff, Homotopy associativity of H-spaces, I and II, Trans. Amer. Math. Soc. 108 (1963), 275-292, 293-312. MR 28:1623

[23] — H-spaces from a homotopy point of view, Lecture Notes in Math. 161, SpringerVerlag, 1970. MR 42:5261

[24] M. Sugawara, A condition that a space is group-like, Math. J. Okayama Univ. 7 (1957), 123-149. MR 20:3546

[25] _ On the homotopy commutativity of groups and loop spaces, Mem. College Sci. Univ. Kyoto Ser. A 33 (1960), 257-269. MR 22:11394

[26] F. D. Williams, Higher homotopy-commutativity, Trans. Amer. Math. Soc. 139 (1969), 191206. MR 39:2163

[27] - Higher homotopy commutativity and extension of maps, Proc. Amer. Math. Soc. 26 (1970), 664-670. MR 42:8491

[28] — Higher Samelson products, J. Pure Appl. Algebra 2 (1972), 249-260. MR 46:2670

[29] G. M. Ziegler, Lectures on Polytopes, Graduate Texts in Math. 152, Springer-Verlag, 1994. MR 96a:52011

Department of Mathematics, Faculty of Science, Kochi University, Kochi 780-8520, JAPAN

E-mail address: hemmi@math.kochi-u.ac.jp

Department of Mathematics, National Defense Academy, Yokosuka 239-8686, Japan

E-mail address: yusuke@nda.ac.jp 